\title{
The Presence of Periodontal Pathogens in Gastric Cancer
}

\author{
Marcel A. de Leeuw \& Manuel X. Duval, GeneCreek
}

\section{Contents}

Introduction

Materials \& Methods Data analysis

\section{Results}

Pathogens in gastric mucosa.. .2

Gastric mucosa community analysis .... 3

Anatomical locations .......... 3

Disease progress ............ . 3

Sample disease location . . . . . . . . . 3

Species relevant in GC . . . . . . . . . . . 4

Prevalence differences . . . . . . . . . 4

Comparison with colorectal cancer . . . . . 4

Eradication therapy ........... 5

Modulation of the gastric mucosa microbiome ........... 5

Discussion 6

Prospects 8

Conclusions $\quad 8$

Declarations 8

Bibliography $\quad 8$

\section{List of Figures}

1 Distribution of prevalence and relative abundance of pathogens in healthy individuals ....... 2

2 Disease status discriminating species . . . . . . . 4

3 Co-exclusion by and co-occurrence with QPS species of GC associated species . . . . . . . . 5

\section{List of Tables}

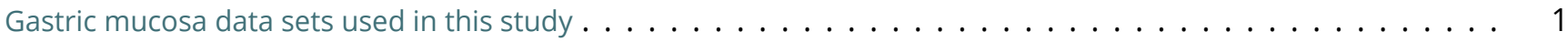

Colorectal cancer biopsy samples used in this study . . . . . . . . . . . . . . . . . . . . . 2

Distribution of periodontal and other pathogens and nitrosating bacteria over community types . . . . . . . 3

Classification results on the disease stage evaluation subset, data set SRP070925 . . . . . . . . . . . . . 3

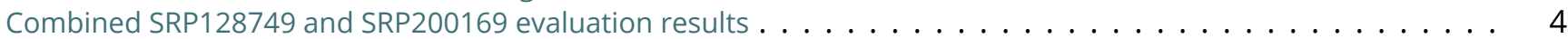

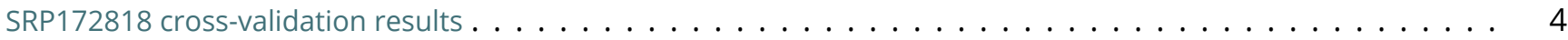

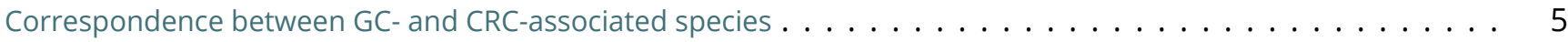

prevalence differences between before and after Hp eradication, SRP165213 . . . . . . . . . . . . . . . 6 
bioRxiv preprint doi: https://doi.org/10.1101/2020.03.23.003426; this version posted April 18, 2020. The copyright holder for this preprint (which was not certified by peer review) is the author/funder, who has granted bioRxiv a license to display the preprint in perpetuity. It is made Revised manuscript available under aCC-BY-NC-ND 4.0 International license.

\title{
The Presence of Periodontal Pathogens in Gastric Cancer
}

\author{
Marcel A. de Leeuw \& Manuel X. Duval, GeneCreek
}

Background. The microbiome is thought to play a role in the development of gastric cancer (GC). Several studies have put forward putatively carcinogenic species in addition to Helicobacter pylori, but are not in perfect alignment, possibly due to variable parameters in the experiments, including downstream processing. Meta-analyses have not been published so far, so there is lack in clinical guidance beyond $H$. pylori eradication therapy.

Methods. Here, we analysed gastric mucosa samples from nine public data sets, including GC samples. Using both unsupervised and supervised learning, we defined fine grain bacterial networks of gastric mucosa and identified species associated with tumor status of samples.

Results. We found anatomic locations and cohort regions among the possible factors leading to the observation of study specific gastric microbiomes. Despite this variability, the periodontal species Fusobacterium nucleatum, Parvimonas micra and Peptostreptococcus stomatis were found in association with tumor status in several datasets. The three species were predicted to be in interaction by ecological network analysis and also formed the intersection of tumor associated species between four GC data sets and five colorectal cancer (CRC) data sets we reanalyzed. We formulated a probiotic composition putatively competing with the GC pathogen spectrum, from correlation analysis in a large superset of gut samples $(n=17,800)$ from clinical- and crowd-sourced studies.

Implications. The overlapping bacterial pathogen spectrum between two gastrointestinal tumor types, GC and $\mathrm{CRC}$, has implications for etiology, treatment and prevention. In vitro testing results reported in literature suggest $H$. pylori eradication treatment should be efficient against the GC pathogen spectrum, yet the existence of an upstream periodontal reservoir is of concern. To address this, we propose longer term use of the formulated probiotics composition.

\section{Introduction}

Gastric cancer (GC) is the sixth most common cancer in the world, with more than $70 \%$ of cases occurring in the developing world. GC is the third leading cause of cancer death worldwide (source: WHO, 2018). More than $50 \%$ of cases occur in Eastern Asia. In Asia, GC is the third most common cancer after breast and lung and is the second most common cause of cancer death after lung cancer [Rahman et al. 2014].

The seroprevalence of Helicobacter pylori is closely related to the incidence of GC [Kato et al. 2004, Ferreccio et al. 2007, Shiota et al. 2013]. In recent years, other bacteria have been proposed as risk factors for GC, including Propionibacterium acnes and Prevotella copri [Gunathilake et al. 2019], Fusobacterium nucleatum [Yamamura et al. 2017, Hsieh et al. 2018] and Leptotrichia wadei [Yang et al. 2016]. Prevotella melaninogenica, Streptococcus anginosus and $P$. acnes have been reported in creased in the tumoral microhabitat [Liu et al. 2019]. The centrality of Peptostreptococcus stomatis, S. anginosus, Parvimonas micra, Slackia exigua and Dialister pneumosintes in GC tissue has been reported [Coker et al. 2018]. P. acnes has also been associated with lymphocytic gastritis [Montalban-Arques et al. 2016].

The availability of a number of these studies in the form of raw microbiome sequence reads offers the possibility to revisit the GC microbiome using a uniform bioinformatics approach, obtain a consensus of additional species possibly involved in GC and address ther- apeutic options beyond H. pylori eradication therapy.

\section{Materials \& Methods}

We identified a total of nine eligible datasets from literature and the NCBI BioProject repository. Exclusion criteria comprised the absence of quality data in the submission and the absence or mismatch of paired end sequences as submitted. Most eligible data sets are from China, Table 1. Scientific publication has been issued for the following projects: PRJEB21497 [Yap et al. 2016], PRJEB21104 [Parsons et al. 2017], PRJEB22107 [Klymiuk et al. 2017], PRJNA428883 [Liu et al. 2019] and PRJNA495436 [He et al. 2019]. For the purpose of comparison, we also included all five colorectal cancer (CRC) data sets we had previously analyzed, Table 2 .

Table 1: Gastric mucosa data sets used in this study. n: number of samples used, 16S: variable regions covered.

\begin{tabular}{llrll}
\hline BioProject & SRA & $\mathrm{n}$ & 16S & region \\
\hline PRJEB21104 & ERP023334 & 121 & V1-V2 & U.K. \\
PRJEB21497 & ERP023753 & 34 & V4 & Malaysia \\
PRJEB22107 & ERP024440 & 32 & V1-V2 & Austria \\
PRJNA313391 & SRP070925 & 119 & V3-V4 & China, Qingdao \\
PRJNA428883 & SRP128749 & 669 & V3-V4 & China, Zhejiang \\
PRJNA481413 & SRP154244 & 397 & V4 & China, Nanchang \\
PRJNA495436 & SRP165213 & 32 & V3-V4 & China, Nanchang \\
PRJNA508819 & SRP172818 & 173 & V3-V4 & China, Zhejiang \\
PRJNA545207 & SRP200169 & 63 & V3-V4 & China, Nanchang \\
total & & 1,544 & & \\
\hline
\end{tabular}


bioRxiv preprint doi: https://doi.org/10.1101/2020.03.23.003426; this version posted April 18, 2020. The copyright holder for this preprint (which was not certified by peer review) is the author/funder, who has granted bioRxiv a license to display the preprint in perpetuity. It is made Revised manuscript available under aCC-BY-NC-ND 4.0 International license.

Table 2: Colorectal cancer biopsy samples used in this study. n: number of samples used, 16S: variable regions covered.

\begin{tabular}{llrll}
\hline BioProject & SRA & $\mathrm{n}$ & 16S & region \\
\hline PRJEB6070 & ERP005534 & 96 & V4 & Germany \\
PRJNA298957 & SRP064975 & 98 & V3-V4 & China, Shanghai \\
PRJNA325650 & SRP076561 & 50 & V3-V4 & Malaysia \\
PRJNA404030 & SRP117763 & 29 & V3-V4 & New Zealand \\
PRJNA445346 & SRP137015 & 211 & V3-V5 & U.S.A. \\
total & & 484 & & \\
\hline
\end{tabular}

\section{Data analysis}

Amplicon Sequence Variants (ASVs) were generated with the R Bioconductor package dada2, version 1.12.1 with recommended parameters [McMurdie, Paul J et al. 2016], involving quality trimming, discarding of sequences with N's, assembly of forward and reverse sequences and contamination and chimera removal. ASVs per data set were subject to further analysis, involving multiple alignment with mafft, version 6.603b [Katoh et al. 2009] and approximatelymaximum-likelihood phylogenetic tree generation with FastTreeMP, version 2.1.11 [Price, Morgan $\mathrm{N}$ et al. 2010], both with default settings.

Taxonomic classification of ASVs were performed by cur|sor version 1.00, an in-house Python and $\mathrm{R}$ program using random forest (RF) based supervised learning on RDP release 11.5. The classifier assigns a species or higher level taxonomic identity to each ASV. Resulting classifications are available from the github repository https://github.com/GeneCreek/GC-manuscript in the form of R data objects.

UniFrac distances were computed using the R Bioconductor package phyloseq, version 1.28.0 [McMurdie and Holmes 2013] on raw ASVs. Further analysis used counts and relative abundances summarized at the species level, using the cur/sor provided taxonomic classifications.
Dirichlet Multinomial Mixtures (DMMs) were computed with the $\mathrm{R}$ bioconductor package DirichletMultinomial, version 1.26.0 [Holmes et al. 2012], using default parameters.

Downstream classification was performed using the $\mathrm{R}$ caret package, version 6.0.84, provided rf model. Variable (taxa) importance was estimated using the mean decrease in node impurity. Multiclass area-underthe-curve (AUC) [Hand and Till 2001] was computed by the R package $\mathrm{pROC}$, version 1.15.3.

Ecological networks were computed using inverse covariance with SPIEC-EASI [Kurtz et al. 2015] as incorporated in the R Bioconductor package SpiecEasi, version 1.0.7, using default parameters.

For the nitrosating status of species, we required that at least one non-redundant genome for the species carries a UniProt annotated nitrate reductase alpha unit gene (narG) [Calmels et al. 1988].

Co-exclusion and co-occurrence between species for probiotics composition were computed using $\chi^{2}$ testing on detectable presence of species in samples $(n=17,800)$ form a proprietary superset of over 40 clinical- and crowd sourced 165 studies, all performed on the Illumina platform.

\section{Results}

\section{Pathogens in gastric mucosa}

Among the species with highest prevalence in gastric mucosa of healthy individuals $(n=85)$, we found a substantial number of opportunistic pathogens, with the majority being known as periodontal pathogens. Figure 1 depicts the distribution of prevalence and relative abundances of the top 20 periodontal and other pathogens. Whereas the position of $H$. pylori is obviously not a surprise, the $60 \%$ prevalence of the skin pathogen $P$. acnes (recently renamed to Cutibacterium acnes) is unexpected. The position of $F$. nucleatum

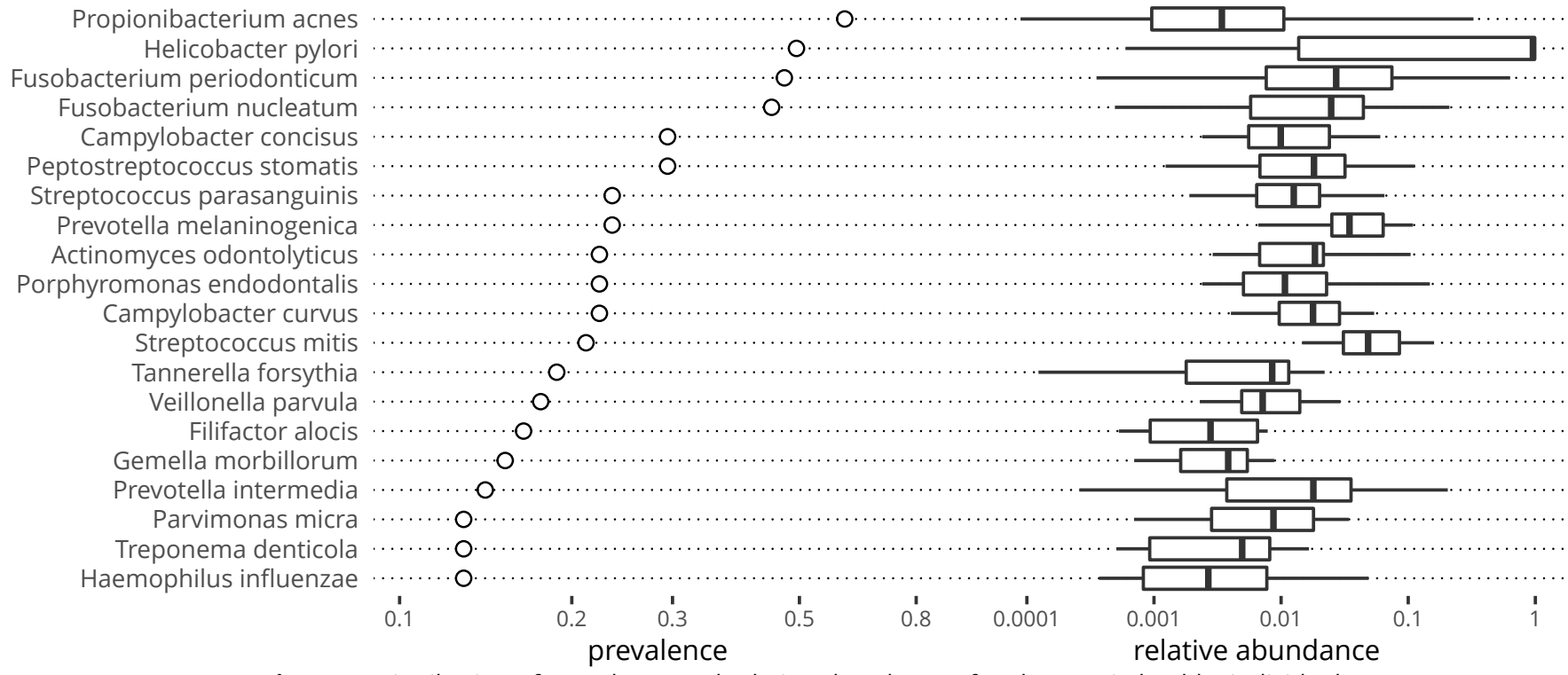

Figure 1: Distribution of prevalence and relative abundance of pathogens in healthy individuals. 
among the top four pathogens is also remarkable. We found 17 distinct ASVs assigned to $P$. acnes and 53 distinct ASVs assigned to $F$. nucleatum in this dataset.

\section{Gastric mucosa community analysis}

We applied unsupervised clustering to investigate microbial gastric mucosa community structure, irrespective of sample disease status. In brief, using Dirichlet Multinomial Mixtures, we obtained an optimal goodness of fit at $k=5$ communities according to the Laplace and AIC evaluations, supplemental Fig. S1. Assigning per sample community types accordingly, we then retrieved the top 100 most important species. We assigned species to community types by maximum contribution. Putative interactions between these species were retrieved from the SPIEC-EASI ecological network constructor, which operated independently from the community structure on all 1,544 samples. Figure S2 depicts the correspondence between species community types and the correlation network.

Table 3: Distribution of periodontal and other pathogens and nitrosating bacteria over community types.

\begin{tabular}{lrrr}
\hline comm. type & periodontal & other & nitrosating \\
\hline dmm 1 & 3 & & 2 \\
dmm 2 & & 1 & \\
dmm 3 & 3 & 9 \\
dmm 4 & 20 & 5 & 8 \\
dmm 5 & & 2 & 1 \\
\hline
\end{tabular}

For community types one and two the dominating species was Helicobacter pylori, with levels exceeding $50 \%$, Fig. S3. Community type two had the lowest phylogenetic diversity of all community types, Fig. S4. Community type four received the majority of periodontal pathogens, whereas community types three and four harbored the most abundant nitrosating species, Table 3.

\section{Anatomical locations}

Data set SRP154244 presents samples from different anatomical gastric locations in patients with gastritis, intestinal metaplasia and gastric cancer. We investigated if microbial signatures cluster by gastric location using random forest (RF) models and ecological networks, Table S3 and Fig. S5. Although we observed segregation between interacting antral curvature species on the one hand and corpus/antrum species on the other hand, it does not seem we can explain the distribution of data sets over the community types by difference in anatomical location alone.

\section{Disease progress}

Data set SRP070925 contains gastric mucosa samples $(n=119)$ from patients with gastritis, intestinal metaplasia, early gastric cancer and advanced gastric cancer. We combined this data set with data set SRP200169, containing gastric mucosa samples $(n=63)$ from healthy subjects. Both are from Chinese cohorts and have been analysed using the $16 \mathrm{~S}$ variable regions V3-V4 combined on the Illumina MiSeq. Performing multidimensional scaling on unweighted UniFrac distances, we found the disease stages are well separated, Fig. S6.

We performed supervised learning of disease progress status with random forests on two thirds of the combined data set, with evaluation on the remaining third. Relative abundances summarized at the species level were used as the analysis substrate. Table 4 provides the classification results. Metaplasia were confounded with gastritis and early cancer, whereas advanced cancer samples were in part classified as early cancer. Healthy, gastritis and early cancer samples were well classified, resulting in an overall multiclass AUC of 0.936.

Table 4: Classification results on the disease stage evaluation subset, data set SRP070925. Predictions are in columns. Multiclass AUC:0.936.

\begin{tabular}{|c|c|c|c|c|c|}
\hline stage & healthy & gastritis & $\begin{array}{l}\text { meta- } \\
\text { plasia }\end{array}$ & $\begin{array}{r}\text { early } \\
\text { cancer }\end{array}$ & $\begin{array}{r}\text { adv. } \\
\text { cancer }\end{array}$ \\
\hline healthy & 22 & & & & \\
\hline gastritis & & 10 & & & \\
\hline metaplasia & & 4 & 2 & 3 & \\
\hline $\begin{array}{l}\text { early } \\
\text { cancer }\end{array}$ & & & & 7 & 1 \\
\hline $\begin{array}{l}\text { advanced } \\
\text { cancer }\end{array}$ & & & & 5 & 7 \\
\hline
\end{tabular}

\section{Sample disease location}

Data set SRP128749 contains gastric mucosa samples $(n=669)$ from GC patients and comprises triplet tumor, peripherical and normal samples. We added biopsies from healthy subjects to this cohort, again using data set SRP200169, to challenge the idea that GC normal reflects entirely healthy tissue. Performing multidimensional scaling on unweighted UniFrac distances, we found the disease locations show interesting separation, Fig. S9. We performed two supervised learning experiments on the combined data set, one with a twothirds training, one-third evaluation setup and a second using one additional data set SRP172818 $(n=173)$ also containing triplets as the cross-validation set. All three data sets are from Chinese cohorts and have been analysed using the $16 \mathrm{~S}$ variable regions V3-V4 combined on the Illumina MiSeq.

Table 5 provides the classification results on the combined SRP128749 and SRP200169 data set. The model performs with a multi-class AUC of 0.842 . Just one normal sample is confounded with healthy samples. The model performance increased to an AUC of 0.906 when trained on the whole combined data set and cross-validated on the SRP172818 data set, Table 6. None of the GC normal samples were confounded with samples from healthy donors. 
Table 5: Combined SRP128749 and SRP200169 evaluation results. Predictions are in columns. Multiclass AUC:0.842

\begin{tabular}{lrrrr}
\hline status & healthy & normal & peripherical & tumor \\
\hline healthy & 22 & & 2 & 4 \\
normal & 1 & 37 & 20 & 11 \\
peripherical & 3 & 10 & 35 & 20 \\
tumor & & 11 & 23 & 46 \\
\hline
\end{tabular}

Table 6: SRP172818 cross-validation results. Predictions are in columns. Multiclass AUC:0.906

\begin{tabular}{|c|c|c|c|c|}
\hline status & healthy & normal & peripherical & tumor \\
\hline \multicolumn{5}{|l|}{ healthy } \\
\hline normal & & 45 & 8 & 4 \\
\hline peripherical & & 7 & 41 & 9 \\
\hline tumor & & 4 & 7 & 48 \\
\hline
\end{tabular}

\section{Species relevant in GC}

We disposed of four data sets having the metadata required for the association of species with tumor status, whether from a disease progress or disease location standpoint. In brief, we processed data sets individually and retrieved the top 50 differentiating species from the random forest models, trained on the data set as a whole. We generates ecological networks using these top species, retaining only connected nodes for display.

Figure 2 provides the putative interaction network of the disease location data sets SRP172818 and SRP128749, showing reproducible tumor association of, and possible interaction between, the oral species $F$. nu-

a

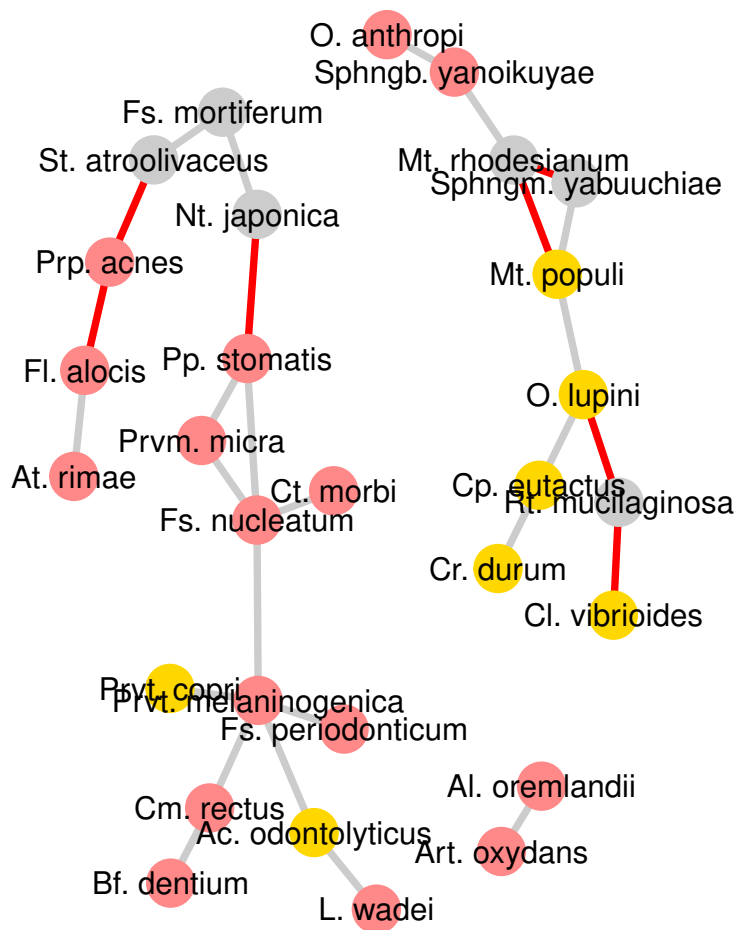

cleatum, P. micra, P. stomatis and Catonella morbi. Correlation indicates the interaction would be cooperative.

Supplemental Fig. S10 and Fig. S11 provide the same analysis for the disease progress data sets SRP070925 and ERP023334, respectively, in the first of which we found $P$. melaninogenica associated with advanced cancer status and in the second $F$. nucleatum with cancer status.

\section{Prevalence differences}

An alternative take on the species differentiating between disease states, using $\chi^{2}$ testing of difference in prevalence, is presented in Tables S4-S8. P. acnes is reproducibly found at over $61 \%$ in GC tumor samples, whereas $P$. stomatis is found at over $54 \%, P$. micra over $37 \%$ and $F$. nucleatum over $35 \%$ in GC tumor samples. The presence of all four roughly doubled over their baseline prevalence in normal samples, Tables S4 and S5.

\section{Comparison with colorectal cancer}

We tested five previously analyzed colorectal cancer (CRC) data sets for presence and interactions of $F$. nucleatum, $P$. micra and $P$. stomatis. All five data sets SRP117763 ( $n=34$, tumor-only) [Purcell et al. 2017], SRP137015 ( $n=211$, tumor/peripherical/normal) [Hale et al. 2018b;a], SRP076561 ( $n=50$, tumor/normal) [Drewes et al. 2017], ERP005534 ( $n=96$, tumor/normal) [Zeller et al. 2014] and SRP064975 ( $n=98$, tumor/peripherical/normal) [Lu et al. 2016] have been subject to publication. We found $F$. nucleatum in inter-

b

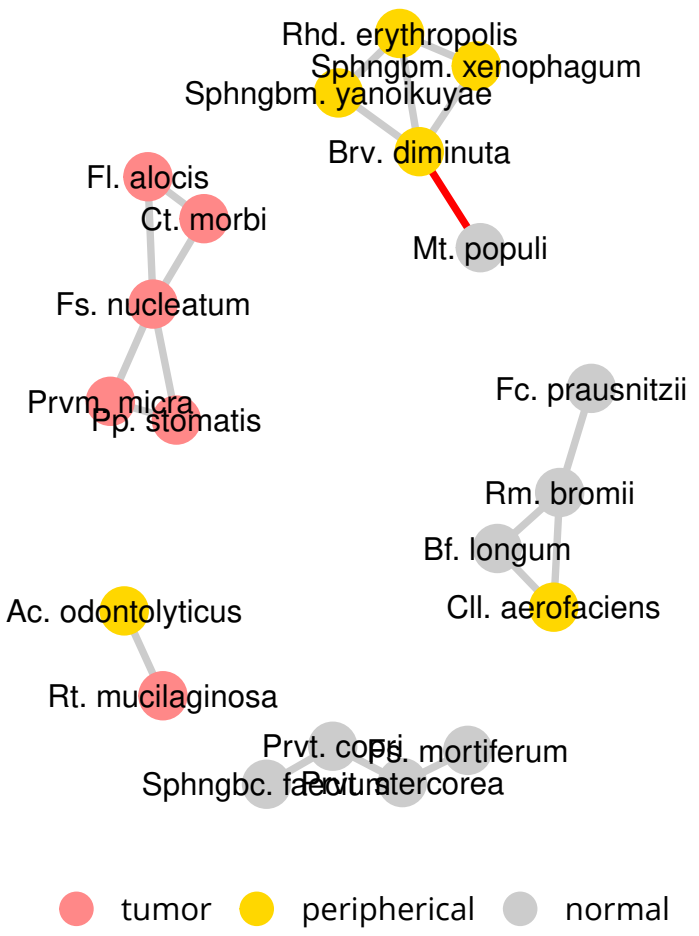

Figure 2: Disease status discriminating species. Data sets a) SRP172818 and b) SRP128749. Only species with interactions are displayed. Location associations are based on maximum mean relative abundance. Co-exclusion is indicated in red. 
bioRxiv preprint doi: https://doi.org/10.1101/2020.03.23.003426; this version posted April 18, 2020. The copyright holder for this preprint (which was not certified by peer review) is the author/funder, who has granted bioRxiv a license to display the preprint in perpetuity. It is made Revised manuscript available under aCC-BY-NC-ND 4.0 International license.

action with $P$. stomatis in SRP137015 and $P$. micra in interaction with $P$. stomatis in data sets SRP117763 and SRP076561, Fig. S12. Prevalence of $F$. nucleatum was found at $70 \%$ or more in tumor samples in SRP117763, Table S8, at 48\% in tumor samples in SRP137015, Table S9 and at 73\% in tumor samples in SRP076561, Table S10. Listing the most abundant cancer associated species in GC and CRC, the intersection between the two cancer types was formed by F. nucleatum, P. micra and $P$. stomatis, Table 7.

Table 7: Correspondence between GC- and CRC-associated species. Numbers reflect the number of datasets in which the species is found associated, out of four possible. Species found in more than one dataset and with relative abundance $>0.5 \%$ in cancer are listed.

\begin{tabular}{lrr}
\hline species & GC & CRC \\
\hline Bacteroides fragilis & & 2 \\
Bacteroides ovatus & & 3 \\
Brevundimonas vesicularis & 2 & \\
Escherichia coli & & 2 \\
Fusobacterium nucleatum & 3 & 3 \\
Gemella morbillorum & & 3 \\
Parvimonas micra & 2 & 3 \\
Peptostreptococcus stomatis & 2 & 2 \\
Prevotella intermedia & & 2 \\
Propionibacterium acnes & 2 & \\
\hline
\end{tabular}

\section{Eradication therapy}

Data set SRP165213 provides mucosa samples, preand post bismuth quadruple $H$. pylori eradication therapy. Using $\chi^{2}$ testing of difference in prevalence, we found several bacteria, including the expected $H$. pylori, exhibit an important drop in prevalence, Table 8. $P$. stomatis, $P$. micra and $F$. nucleatum on the other hand showed a moderately significant prevalence increase.

\section{Modulation of the gastric mucosa microbiome}

Using prevalence data from 17,800 gut samples, including the samples used in this study, we probed for qualified presumption of safety (QPS) species found in co-exclusion with the species of interest panel identified above. Figure 3 shows the result. Bifidobacterium longum appears as the most promising QPS species, followed by Streptococcus salivarius both of which are being used in probiotic products and are actually detectable in gastric mucosa samples, see Fig. $3 b$ for $B$. longum. In the healthy data set SRP200169 we found 27 ASVs for B. longum but none for S. salivarius, indicating that the latter is probably not commensal in the stomach in healthy individuals.

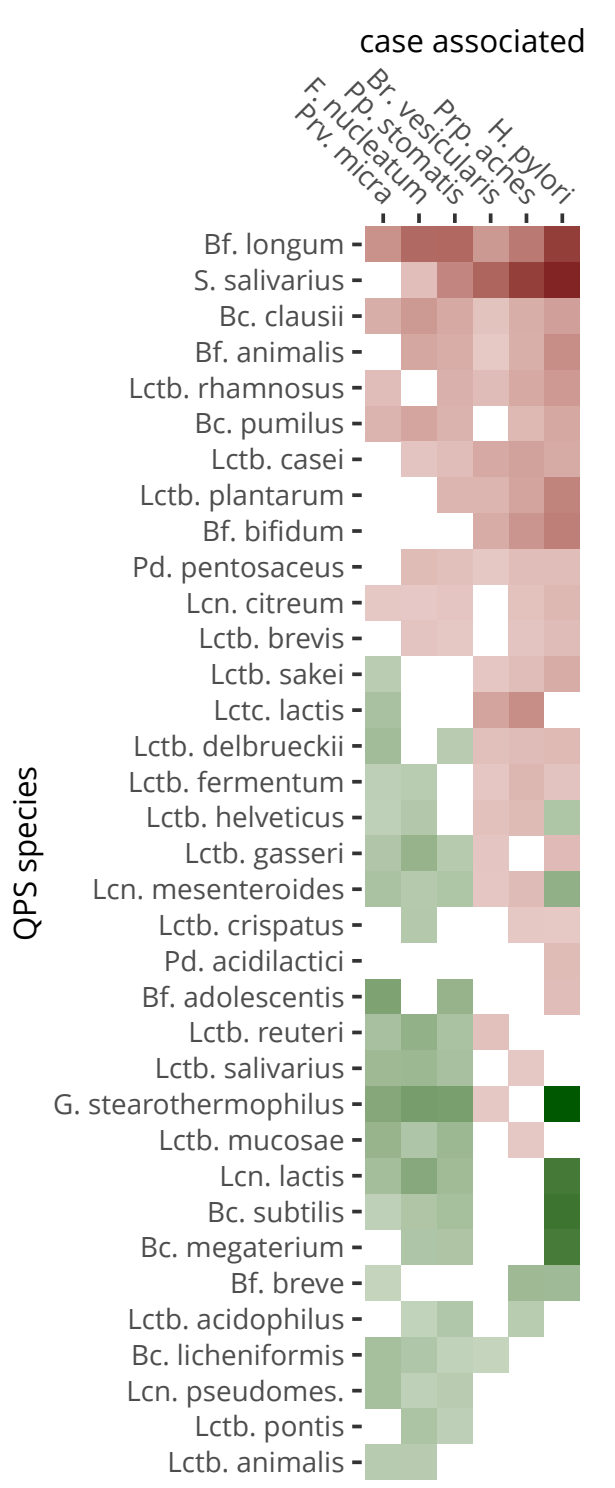

Figure 3: Co-exclusion by and co-occurrence with QPS species of GC associated species. Putative inhibition is in shades of red, potential synergy in shades of green. White reflect neutrality or too little combined prevalence to make a call. Genera are abbreviated as follows: Bcl.: Bacillus, Bf.: Bifidobacterium, Gb.: Geobacillus, Lcn.: Leuconostic, Lctb.: Lactobacillus, Lctc.: Lactococcus, Pd.: Pediococcus, S.: Streptococcus. 
bioRxiv preprint doi: https://doi.org/10.1101/2020.03.23.003426; this version posted April 18, 2020. The copyright holder for this preprint (which was not certified by peer review) is the author/funder, who has granted bioRxiv a license to display the preprint in perpetuity. It is made Revised manuscript available under aCC-BY-NC-ND 4.0 International license.

Table 8: prevalence differences between before and after Hp eradication, SRP165213.

\begin{tabular}{|c|c|c|c|c|c|c|}
\hline species & association & pvalue & & before & after & count \\
\hline Helicobacter pylori & before & $3.8 \mathrm{e}-06$ & $\star \star \star \star$ & $17 / 17(100.0 \%)$ & $2 / 15(13.3 \%)$ & 19 \\
\hline Brevundimonas diminuta & before & $1.7 e-05$ & 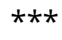 & $17 / 17(100.0 \%)$ & $3 / 15(20.0 \%)$ & 20 \\
\hline Sphingobium yanoikuyae & before & $1.3 e-03$ & *夫 & $13 / 17(76.5 \%)$ & $2 / 15(13.3 \%)$ & 15 \\
\hline Sphingomonas yabuuchiae & before & $4.6 e-03$ & ** & $13 / 17(76.5 \%)$ & $3 / 15(20.0 \%)$ & 16 \\
\hline Sphingobium xenophagum & before & $9.5 e-03$ & ** & $11 / 17(64.7 \%)$ & $2 / 15(13.3 \%)$ & 13 \\
\hline Propionibacterium acnes & before & $1.0 e+00$ & & $14 / 17(82.4 \%)$ & $12 / 15(80.0 \%)$ & 26 \\
\hline Bifidobacterium adolescentis & after & $1.0 \mathrm{e}-04$ & $\star \star \star$ & $2 / 17(11.8 \%)$ & $13 / 15(86.7 \%)$ & 15 \\
\hline Ruminococcus bromii & after & 3.0e-04 & $\star \star *$ & $4 / 17(23.5 \%)$ & $14 / 15(93.3 \%)$ & 18 \\
\hline Dorea longicatena & after & $3.6 e-04$ & $\star \star \star$ & $1 / 17(5.9 \%)$ & $11 / 15(73.3 \%)$ & 12 \\
\hline Leptotrichia wadei & after & $5.8 e-03$ & ** & $0 / 17(0.0 \%)$ & $7 / 15(46.7 \%)$ & 7 \\
\hline Peptostreptococcus stomatis & after & $3.4 \mathrm{e}-02$ & * & $5 / 17(29.4 \%)$ & $11 / 15(73.3 \%)$ & 16 \\
\hline Parvimonas micra & after & $8.2 \mathrm{e}-02$ & & $0 / 17(0.0 \%)$ & $4 / 15(26.7 \%)$ & 4 \\
\hline Fusobacterium nucleatum & after & $5.2 \mathrm{e}-01$ & & $5 / 17(29.4 \%)$ & $7 / 15(46.7 \%)$ & 12 \\
\hline
\end{tabular}

\section{Discussion}

In this above, we revisited public gastric mucosa and colorectal cancer data sets, taking into account recent advances in processing of amplicon metagenomic sequences [Callahan et al.2017], establishing species level taxonomic classification.

Limitations. Use of a healthy cohort analyzed as a separate batch and from a different regional cohort does not allow to control for batch- or regional effects in supervised learning. Regional clustering of GC microbiota has been reported previously [Yu et al. 2017]. So our case that samples from healthy donors are distinct from GC normal samples in GC patients is a delicate case. For confirmation of this hypothesis, healthy donors need to be recruited from the same population as the GC patients.

Four subspecies are known for F. nucleatum. Our taxonomic classifier does not resolve down to the level of subspecies, so all counts and relative abundances for $F$. nucleatum may conceal different subspecies, moreover so since in CRC, multiple subspecies have been isolated from biopsies [Brennan and Garrett 2019] and since we detected several tens of distinct ASVs associated with $F$. nucleatum.

Low biomass and contamination. P. acnes has been proposed as a possible contaminant of many experiments [Mollerup et al. 2016]. This is particularly relevant for gastric samples which are of low biomass as compared to biopsies from the lower Gl tract. That does not mean we need to discard the bacterium altogether, notably not if it shows significant increase in tumor sample locations as in data sets SRP172818 and SRP128749, but it could mean its baseline presence is overestimated and hence its status as a gastric mucosa commensal [Delgado et al. 2011]. Its position as a prevalent but low abundant species in healthy subjects gives credit to the contamination thesis. The number of ASVs associated with $P$. acnes though suggests that if there is contamination, it originates from multiple individuals. The fact that the bacterium never reached high abundance in the experiments means that it did not contaminate low biomass samples in particular.

Helicobacter pylori. In all data sets, we found gastric mucosa samples completely exempt of $\mathrm{H}$. pylori, including in normal and peripherical samples, which opens the possibility that other pathogens play a role in GC. We did not find $H$. pylori in significant interaction, which is unexpected and discrepant to findings on the same data set SRP128749 reported [Liu et al. 2019]. We attribute this discrepancy to the use of a more stringent ecological network inference [Kurtz et al. 2015]. On the other hand, report has been made that $H$. pylori presence did not affect microbial community composition [Bik et al. 2006]. So it seems that although H. pylori may create oncogenic conditions through host interaction, there does not seem to be a direct benefit or detriment of such conditions for other bacteria.

Cohort specific species. Our results show species found in gastric mucosa have a strong cohort specific distribution of species. Within cohort prediction of sample disease status or location status based on the microbiome composition is performing well with AUCs over 0.8 , so despite its diversity, there is a clear sample status signature in the microbiome composition.

Nitrosating species. Nitrosating bacteria convert nitrogen compounds in gastric fluid to potentially carcinogenic N-nitroso compounds (NOCs), which are believed to contribute to gastric cancer [Sharma et al. 1984, Mowat et al. 2000, Jo et al. 2016, Ferreira et al. 2018, Park et al. 2019]. We found nitrosating bacteria were not uniformly distributed over gastric mucosa community types. Community type four combines nitrosating species with periodontal pathogens and can be considered as the highest GC risk community type.

Periodontal and CRC pathogens. It has been reported that among patients with periodontal disease, high levels of colonization of periodontal pathogens are associated with an increased risk of gastric precancerous lesions [Salazar et al. 2013]. We found the periodontal pathogens $F$. nucleatum, $P$. micra and $P$. stomatis to be 
bioRxiv preprint doi: https://doi.org/10.1101/2020.03.23.003426; this version posted April 18, 2020. The copyright holder for this preprint (which was not certified by peer review) is the author/funder, who has granted bioRxiv a license to display the preprint in perpetuity. It is made Revised manuscript available under aCC-BY-NC-ND 4.0 International license.

commensal but also associated with tumor status and in direct interaction in several data sets. These three species were also found in association with tumor status in CRC data sets revisited and correspond with a CRC subtype with strong immune signature [Purcell et al. 2017]. Revisiting the CRC data sets, we found in part the same interactions as in GC. Two recent meta-analysis of CRC case-control studies placed $F$. nucleatum, P. micra and $P$. stomatis among the top five carcinoma enriched species [Drewes et al. 2017, Wirbel et al. 2019]. F. nucleatum and $P$. stomatis have also been proposed among a panel of species for early detection of CRC [Zeller et al. 2014].

Virulence. The gram negative bacterium F. nucleatum promotes tumor development by inducing inflammation and host immune response in the CRC microenvironment. Its adhesion to the intestinal epithelium can cause the host to produce inflammatory factors and recruit inflammatory cells, creating an environment which favors tumor growth. Treatment of mice bearing a colon cancer xenograft with the antibiotic metronidazole reduced Fusobacterium load, cancer cell proliferation, and overall tumor growth [Bullman et al. 2017]. F. nucleatum can induce immune suppression in gut mucosa, contributing to the progression of CRC [Wu et al. 2019]. In CRC, F. nucleatum is predicted to produce hydrogen sulfide $\left(\mathrm{H}_{2} \mathrm{~S}\right)$ [Hale et al. 2018b], which is a metabolite with a dual role, both carcinogenic and antiinflammatory. Epithelial cells react to F. nucleatum by activation of multiple cell signaling pathways that lead to production of collagenase 3 , increased cell migration, formation of lysosome-related structures, and cell survival [Uitto et al. 2005].

Furthermore, it is predicted $F$. nucleatum infection regulates multiple signaling cascades which could lead to up-regulation of proinflammatory responses, oncogenes, modulation of host immune defense mechanism and suppression of DNA repair system [Kumar et al. 2016]. There does not seem to be a reason why $F$. nucleatum would not be pathogenic in gastric tissue whereas it is in periodontal, respiratory tract, tonsils, appendix, colonic and other tissues [Han 2015].

The gram positive anaerobe $P$. stomatis has been isolated from a variety of periodontal and endodontic infections, as well as infections in other bodyparts [Downes and Wade 2006]. The species has been found associated with oral squamous cell carcinoma (OSCC) [Pushalkar et al. 2012]. At present, little is known about the specifics of its pathogenicity. The type strain (DSM 17678) genome harbors a gene (mprF, phosphatidylglycerol lysyltransferase) producing lysylphosphatidylglycerol (LPG), a major component of the bacterial membrane with a positive net charge. LPG synthesis contributes to bacterial virulence as it is involved in the resistance mechanism against cationic antimicrobial peptides produced by the host's immune system and by competing microorganisms. Contrary to other
Peptostreptococci, P. stomatis does not produce intestinal barrier enforcing indole-3-propionic acid (IPA) or indoleacrylic acid (IA) [Wlodarska et al. 2017].

P. micra, previously known as (Pepto)streptococcus micros, is a gram positive anaerobe which is known to be involved in periodontal infections. It has also been isolated from OSCC [Hooper et al. 2007]. It is a producer of collagenase and of limited elastolytic and hemolytic activity [Ota-Tsuzuki and Alves Mayer 2010]. In a mouse CRC model, P. micra elicited increased Th2 and Th17 cells, decreased Th1 cells and increased inflammation [Yu et al. 2019].

The oral cavity as reservoir. It has been shown that in a number of cases $(6 / 14,43 \%)$ identical $F$. nucleatum strains could be recovered from CRC and saliva of the same patients [Komiya et al. 2019]. Furthermore, the oral microbiome composition is to a certain extent predictive for CRC disease progress status [Flemer et al. 2018]. It is tempting to speculate that a similar relationship could be explored for disease progress in GC.

Biofilm formation. F. nucleatum is regarded as a central organism for dental biofilm maturation due to its wide ability to aggregate with other microorganisms, such as Porphyromonas gingivalis [Tavares et al. 2018]. It is considered as a bridge bacterium between early and late colonizers in dental plaque [He et al. 2016]. The eventuality of $H$. pylori- and non $H$. pylory biofilm formation in the gastric environment has been raised [Rizzato et al. 2019]. Our ecologic interaction networks suggests $F$. nucleatum and other bacteria, but not $H$. pylori, could indeed engage in gastric mucosa biofilms and more particularly in GC biofilms.

Antibiotherapy. Helicobacter pylori eradication therapy has been shown to have a prophylactic effect against GC [Kwok et al. 2008]. The first-line therapy consists of a proton pump inhibitor (PPI) or ranitidine bismuth citrate, with any two antibiotics among amoxicillin, clarithromycin and metronidazole. In vitro testing has shown Peptostreptococcus stomatis is sensitive to amoxicillin and metronidazole [Könönen et al. 2007]. F. nucleatum is sensitive to amoxicillin or amoxicillin/clavulanate combination therapy [Jacinto et al. 2008] and to metronidazole [Shilnikova and Dmitrieva 2015, Bullman et al. 2017]. Parvimonas micra is sensitive to amoxicillin/clavulanate and metronidazole [Veloo et al. 2011]. In vivo sensitivity of the species may differ and in addition, with the oral cavity as a reservoir, periodontal pathogens could recolonize the gastric environment and take advantage of the space cleared by H. pylori, which is what our data suggests.

Probiotics use. We predicted in silico that several QPS species could be effective against the spectrum of $H$. pylori and the periodontal pathogens discussed above. Our findings are coherent with the report that probiotics including Bifidobacterium longum, Lactobacillus acidophilus, and Enterococcus faecalis significantly reduced 
the abundance of $F$. nucleatum in CRC surgery patients by nearly 5 -fold, whilst normalizing dysbiosis [Gao et al. 2015]. In vitro adhesion inhibition of gram-negative species by $B$. longum has been reported [Inturri et al. 2016]. Other than adhesion inhibitors, bifidobacteria produce acetate and lactate as well as vitamins, antioxidants, polyphenols, and conjugated linoleic acids which have been proposed to act as chemical barrier against pathogen proliferation [Inturri et al. 2019]. Streptococcus salivarius not only inhibits adhesion of pathogens to epithelial cells, but also produces bacteriocins [Manning et al. 2016].

\section{Prospects}

In future GC microbiome studies, it appears imperative to include normal controls from healthy subjects so that normal samples from GC patients can be properly compared against samples from healthy subjects. Fluorescent in situ hybridization could be used in case of gastrectomy to confirm biofilm status of the aforementioned pathogen spectrum. A long term maintenance formula using probiotics after an antibiotics eradication course can be of interest as a treatment option. A variety of Bifidobacterium longum strains are used in several probiotic preparations commercially available whereas Streptococcis salivarius strain K12 [Burton et al. 2006] is also commercially available.

\section{Conclusions}

In conclusion, we found disease progress and sample disease status is not reflected in the overall bacterial community type of mucosa. Rather, community types are populated by potentially regionally distinct species. Despite this diversity, we found periodontal pathogens as a common denomicator. These pathogens were also identified in CRC, establishing possible microbial similarities between subtypes of GC and CRC, with implications for etiology, treatment and prevention. Correlation networks suggest these species, as in dental plaque and in CRC, engage in biofilm formation in gastric mucosa. Probiotics should be considered as a treatment option, after $\mathrm{H}$. pylori eradication therapy, to avoid recolonization by periodontal pathogens.

\section{Declarations}

Acknowledgements. The authors received no financial support for this study. The authors acknowledge the contributions to the Short Read Archive made by the respective institutions and acknowledge scientific journals for enforcing this practice.

Conflict of interest. The authors have no conflict of interests related to this publication.

Authors' contributions. Study design, data collection, data analysis and writing of the manuscript (ML); data analysis and writing of the manuscript (MD).

\section{Bibliography}

Elisabeth M Bik, Paul B Eckburg, Steven R Gill, Karen E Nelson, Elizabeth A Purdom, Fritz Francois, Guillermo Perez-Perez, Martin J Blaser, and David A Relman. Molecular analysis of the bacterial microbiota in the human stomach. PNAS, 103(3):732-737, January 2006. doi: 10.1073/pnas.0506655103.

Caitlin A Brennan and Wendy S Garrett. Fusobacterium nucleatum - symbiont, opportunist and oncobacterium. Nature reviews. Microbiology, 17(3):156166, March 2019. doi: 10.1038/s41579-018-0129-6.

Susan Bullman, Chandra S Pedamallu, Ewa Sicinska, Thomas E Clancy, Xiaoyang Zhang, Diana Cai, Donna Neuberg, Katherine Huang, Fatima Guevara, Timothy Nelson, Otari Chipashvili, Timothy Hagan, Mark Walker, Aruna Ramachandran, Begoña Diosdado, Garazi Serna, Nuria Mulet, Stefania Landolfi, Santiago Ramon Y Cajal, Roberta Fasani, Andrew J Aguirre, Kimmie Ng, Elena Élez, Shuji Ogino, Josep Tabernero, Charles S Fuchs, William C Hahn, Paolo Nuciforo, and Matthew Meyerson. Analysis of Fusobacterium persistence and antibiotic response in colorectal cancer. Science, 358(6369):1443-1448, December 2017. doi: 10.1126/science.aal5240.

Jeremy P Burton, Philip A Wescombe, Chris J Moore, Chris N Chilcott, and John R Tagg. Safety assessment of the oral cavity probiotic Streptococcus salivarius K12. Applied and Environmental Microbiology, 72(4):3050-3053, April 2006. doi: 10.1128/AEM.72.4. 3050-3053.2006.

Benjamin J Callahan, Paul J McMurdie, and Susan P Holmes. Exact sequence variants should replace operational taxonomic units in marker-gene data analysis. The ISME Journal, 11(12):2639-2643, December 2017. doi: 10.1038/ismej.2017.119.

$\mathrm{S}$ Calmels, H Ohshima, and H Bartsch. Nitrosamine formation by denitrifying and non-denitrifying bacteria: implication of nitrite reductase and nitrate reductase in nitrosation catalysis. Journal of general microbiology, 134(1):221-226, January 1988. doi: 10.1099/ 00221287-134-1-221.

Olabisi Oluwabukola Coker, Zhenwei Dai, Yongzhan Nie, Guijun Zhao, Lei Cao, Geicho Nakatsu, William Kk Wu, Sunny Hei Wong, Zigui Chen, Joseph J Y Sung, and Jun Yu. Mucosal microbiome dysbiosis in gastric carcinogenesis. Gut, 67(6):1024-1032, June 2018. doi: 10.1136/gutjnl-2017-314281.

Susana Delgado, Adolfo Suárez, and Baltasar Mayo. Identification, typing and characterisation of Propionibacterium strains from healthy mucosa of the human stomach. International Journal of Food Microbi- 
bioRxiv preprint doi: https://doi.org/10.1101/2020.03.23.003426; this version posted April 18, 2020. The copyright holder for this preprint (which was not certified by peer review) is the author/funder, who has granted bioRxiv a license to display the preprint in perpetuity. It is made Revised manuscript available under aCC-BY-NC-ND 4.0 International license.

ology, 149(1):65-72, September 2011. doi: 10.1016/j. ijfoodmicro.2011.01.028.

Julia Downes and William G Wade. Peptostreptococcus stomatis sp. nov., isolated from the human oral cavity. International Journal of Systematic and Evolutionary Microbiology, 56(Pt 4):751-754, April 2006. doi: 10.1099/ijs.0.64041-0.

Julia L Drewes, James R White, Christine M Dejea, Payam Fathi, Thevambiga lyadorai, Jamuna Vadivelu, April C Roslani, Elizabeth C Wick, Emmanuel F Mongodin, Mun Fai Loke, Kumar Thulasi, Han Ming Gan, Khean Lee Goh, Hoong Yin Chong, Sandip Kumar, Jane W Wanyiri, and Cynthia L Sears. High-resolution bacterial 16S rRNA gene profile meta-analysis and biofilm status reveal common colorectal cancer consortia. NPJ biofilms and microbiomes, 3(1):34, 2017. doi: 10.1038/s41522-017-0040-3.

Catterina Ferreccio, Antonio Rollán, Paul R Harris, Carolina Serrano, Alessandra Gederlini, Paula Margozzini, Claudia Gonzalez, Ximena Aguilera, Alejandro Venegas, and Alejandro Jara. Gastric cancer is related to early Helicobacter pylori infection in a highprevalence country. Cancer epidemiology, biomarkers \& prevention : a publication of the American Association for Cancer Research, cosponsored by the American Society of Preventive Oncology, 16(4):662-667, April 2007. doi: 10.1158/1055-9965.EPI-06-0514.

Rui M Ferreira, Joana Pereira-Marques, Ines PintoRibeiro, Jose L Costa, Fatima Carneiro, Jose C Machado, and Ceu Figueiredo. Gastric microbial community profiling reveals a dysbiotic cancer-associated microbiota. Gut, 67(2):226-236, February 2018. doi: 10.1136/gutjnl-2017-314205.

Burkhardt Flemer, Ryan D Warren, Maurice P Barrett, Katryna Cisek, Anubhav Das, Ian B Jeffery, Eimear Hurley, Micheal O'Riordain, Fergus Shanahan, and Paul W O'Toole. The oral microbiota in colorectal cancer is distinctive and predictive. Gut, 67(8):1454-1463, August 2018. doi: 10.1136/gutjnl-2017-314814.

Zhiguang Gao, Bomin Guo, Renyuan Gao, Qingchao Zhu, Wen Wu, and Huanlong Qin. Probiotics modify human intestinal mucosa-associated microbiota in patients with colorectal cancer. Molecular medicine reports, 12(4):6119-6127, October 2015. doi: 10.3892/ mmr.2015.4124.

Madhawa Neranjan Gunathilake, Jeonghee Lee, II Ju Choi, Young-II Kim, Yongju Ahn, Chanhyeok Park, and Jeongseon Kim. Association between the relative abundance of gastric microbiota and the risk of gastric cancer: a case-control study. Scientific Reports, 9(1):13589-11, September 2019. doi: 10.1038/ s41598-019-50054-x.
Vanessa L Hale, Patricio Jeraldo, Jun Chen, Michael Mundy, Janet Yao, Sambhawa Priya, Gary Keeney, Kelly Lyke, Jason Ridlon, Bryan A White, Amy J French, Stephen N Thibodeau, Christian Diener, Osbaldo Resendis-Antonio, Jaime Gransee, Tumpa Dutta, Xuan-Mai Petterson, Jaeyun Sung, Ran Blekhman, Lisa Boardman, David Larson, Heidi Nelson, and Nicholas Chia. Distinct microbes, metabolites, and ecologies define the microbiome in deficient and proficient mismatch repair colorectal cancers. Genome Medicine, 10(1):78-13, October 2018a. doi: 10.1186/ s13073-018-0586-6.

Vanessa L Hale, Patricio Jeraldo, Michael Mundy, Janet Yao, Gary Keeney, Nancy Scott, E Heidi Cheek, Jennifer Davidson, Megan Greene, Christine Martinez, John Lehman, Chandra Pettry, Erica Reed, Kelly Lyke, Bryan A White, Christian Diener, Osbaldo ResendisAntonio, Jaime Gransee, Tumpa Dutta, Xuan-Mai Petterson, Lisa Boardman, David Larson, Heidi Nelson, and Nicholas Chia. Synthesis of multi-omic data and community metabolic models reveals insights into the role of hydrogen sulfide in colon cancer. METHODS, 149:59-68, October 2018b. doi: 10.1016/ j.ymeth.2018.04.024.

Yiping W Han. Fusobacterium nucleatum: a commensalturned pathogen. Current opinion in microbiology, 23: 141-147, February 2015. doi: 10.1016/j.mib.2014.11. 013.

David J Hand and Robert J Till. A Simple Generalisation of the Area Under the ROC Curve for Multiple Class Classification Problems. Machine Learning, 45(2):171186, 2001. doi: 10.1023/A:1010920819831.

Cong He, Chao Peng, Huan Wang, Yaobin Ouyang, Zhenhua Zhu, Xu Shu, Yin Zhu, and Nonghua Lu. The eradication of Helicobacter pylori restores rather than disturbs the gastrointestinal microbiota in asymptomatic young adults. Helicobacter, 24(4):e12590, August 2019. doi: 10.1111/hel.12590.

Zhiyan He, Zhengwei Huang, Wei Zhou, Zisheng Tang, Rui Ma, and Jingping Liang. Anti-biofilm Activities from Resveratrol against Fusobacterium nucleatum. Frontiers in Microbiology, 7(159797):1065, 2016. doi: 10.3389/fmicb.2016.01065.

Ian Holmes, Keith Harris, and Christopher Quince. Dirichlet multinomial mixtures: generative models for microbial metagenomics. PLOS ONE, 7(2):e30126, 2012. doi: 10.1371/journal.pone.0030126.

Samuel J Hooper, St-John Crean, Michael J Fardy, Michael A O Lewis, David A Spratt, William G Wade, and Melanie J Wilson. A molecular analysis of the bacteria present within oral squamous cell carcinoma. Journal of medical microbiology, 56(Pt 12):1651-1659, December 2007. doi: 10.1099/jmm.0.46918-0. 
bioRxiv preprint doi: https://doi.org/10.1101/2020.03.23.003426; this version posted April 18, 2020. The copyright holder for this preprint (which was not certified by peer review) is the author/funder, who has granted bioRxiv a license to display the preprint in perpetuity. It is made

Yung-Yu Hsieh, Shui-Yi Tung, Hung-Yu Pan, Chih-Wei Yen, Huang-Wei Xu, Ying-Jhen Lin, Yi-Fang Deng, WanTing Hsu, Cheng-Shyong Wu, and Chin Li. Increased Abundance of Clostridium and Fusobacterium in Gastric Microbiota of Patients with Gastric Cancer in Taiwan. Scientific Reports, 8(1):158-11, January 2018. doi: 10.1038/s41598-017-18596-0.

R Inturri, A Stivala, P M Furneri, and G Blandino. Growth and adhesion to HT-29 cells inhibition of Gram-negatives by Bifidobacterium longum BB536 e Lactobacillus rhamnosus HN001 alone and in combination. European review for medical and pharmacological sciences, 20(23):4943-4949, December 2016.

Rosanna Inturri, Laura Trovato, Giovanni Li Volti, Salvatore Oliveri, and Giovanna Blandino. In vitro inhibitory activity of Bifidobacterium longum BB536 and Lactobacillus rhamnosus HN001 alone or in combination against bacterial and Candida reference strains and clinical isolates. Heliyon, 5(11):e02891, November 2019. doi: 10.1016/j.heliyon.2019.e02891.

Rogério C Jacinto, Francisco Montagner, Fernanda G C Signoretti, Geovania C Almeida, and Brenda P F A Gomes. Frequency, microbial interactions, and antimicrobial susceptibility of Fusobacterium nucleatum and Fusobacterium necrophorum isolated from primary endodontic infections. Journal of endodontics, 34(12):1451-1456, December 2008. doi: 10.1016/ j.joen.2008.08.036.

Hyun Jin Jo, Jaeyeon Kim, Nayoung Kim, Ji Hyun Park, Ryoung Hee Nam, Yeong-Jae Seok, Yeon-Ran Kim, Joo Sung Kim, Jung Mogg Kim, Jung Min Kim, Dong Ho Lee, and Hyun Chae Jung. Analysis of Gastric Microbiota by Pyrosequencing: Minor Role of Bacteria Other Than Helicobacter pylori in the Gastric Carcinogenesis. Helicobacter, 21(5):364-374, October 2016. doi: 10.1111/hel.12293.

M Kato, M Asaka, Y Shimizu, A Nobuta, H Takeda, T Sugiyama, and Multi-Centre Study Group. Relationship between Helicobacter pylori infection and the prevalence, site and histological type of gastric cancer. Alimentary Pharmacology \& Therapeutics, 20 Suppl 1(s1):85-89, July 2004. doi: 10.1111/j.1365-2036.2004. 01987.x.

Kazutaka Katoh, George Asimenos, and Hiroyuki Toh. Multiple alignment of DNA sequences with MAFFT. Methods in molecular biology (Clifton, N.J.), 537(Suppl 5):39-64, 2009. doi: 10.1007/978-1-59745-251-9_3.

Ingeborg Klymiuk, Ceren Bilgilier, Alexander Stadlmann, Jakob Thannesberger, Marie-Theres Kastner, Christoph Högenauer, Andreas Püspök, Susanne Biowski-Frotz, Christiane Schrutka-Kölbl, Gerhard G Thallinger, and Christoph Steininger. The Human Gastric Microbiome Is Predicated upon Infection with He- licobacter pylori. Frontiers in Microbiology, 8:e2836-12, December 2017. doi: 10.3389/fmicb.2017.02508.

Yasuhiko Komiya, Yumi Shimomura, Takuma Higurashi, Yutaka Sugi, Jun Arimoto, Shotaro Umezawa, Shiori Uchiyama, Mitsuharu Matsumoto, and Atsushi Nakajima. Patients with colorectal cancer have identical strains of Fusobacterium nucleatum in their colorectal cancer and oral cavity. Gut, 68(7):1335-1337, July 2019. doi: 10.1136/gutjnl-2018-316661.

Eija Könönen, Anne Bryk, Päivi Niemi, and Arja KanervoNordström. Antimicrobial susceptibilities of Peptostreptococcus anaerobius and the newly described Peptostreptococcus stomatis isolated from various human sources. Antimicrobial agents and chemotherapy, 51(6):2205-2207, June 2007. doi: 10.1128/AAC. 00056-07.

Amit Kumar, Pragna Lakshmi Thotakura, Basant Kumar Tiwary, and Ramadas Krishna. Target identification in Fusobacterium nucleatum by subtractive genomics approach and enrichment analysis of host-pathogen protein-protein interactions. BMC Microbiology, 16(1): 84-12, May 2016. doi: 10.1186/s12866-016-0700-0.

Zachary D Kurtz, Christian L Müller, Emily R Miraldi, Dan R Littman, Martin J Blaser, and Richard A Bonneau. Sparse and compositionally robust inference of microbial ecological networks. PLoS Computational Biology, 11(5):e1004226, May 2015. doi: 10.1371/ journal.pcbi.1004226.

Avelyn Kwok, Thao Lam, Peter Katelaris, and Rupert WI Leong. Helicobacter pylori eradication therapy: indications, efficacy and safety. Expert opinion on drug safety, 7(3):271-281, May 2008. doi: 10.1517/ 14740338.7.3.271.

Xiaosun Liu, Li Shao, Xia Liu, Feng Ji, Ying Mei, Yiwen Cheng, Fengping Liu, Chongxian Yan, Lanjuan Li, and Zongxin Ling. Alterations of gastric mucosal microbiota across different stomach microhabitats in a cohort of 276 patients with gastric cancer. EBioMedicine, 40:336-348, February 2019. doi: 10.1016/j.ebiom. 2018.12.034.

Yingying Lu, Jing Chen, Junyuan Zheng, Guoyong $\mathrm{Hu}$, Jingjing Wang, Chunlan Huang, Lihong Lou, Xingpeng Wang, and Yue Zeng. Mucosal adherent bacterial dysbiosis in patients with colorectal adenomas. Scientific Reports, 6:26337, May 2016. doi: 10.1038/srep26337.

Jayne Manning, Eileen M Dunne, Philip A Wescombe, John D F Hale, E Kim Mulholland, John R Tagg, Roy M Robins-Browne, and Catherine Satzke. Investigation of Streptococcus salivarius-mediated inhibition of pneumococcal adherence to pharyngeal epithelial cells. BMC Microbiology, 16(1):225-9, September 2016. doi: 10.1186/s12866-016-0843-z. 
bioRxiv preprint doi: https://doi.org/10.1101/2020.03.23.003426; this version posted April 18, 2020. The copyright holder for this preprint (which was not certified by peer review) is the author/funder, who has granted bioRxiv a license to display the preprint in perpetuity. It is made Revised manuscript available under aCC-BY-NC-ND 4.0 International license.

Paul J McMurdie and Susan Holmes. phyloseq: an $\mathrm{R}$ package for reproducible interactive analysis and graphics of microbiome census data. PLOS ONE, 8(4): e61217, 2013. doi: 10.1371/journal.pone.0061217.

McMurdie, Paul J, Rosen, Michael J, Han, Andrew W, Johnson, Amy Jo A, Holmes, Susan P, and Callahan, Benjamin J. DADA2: High-resolution sample inference from Illumina amplicon data. Nature Methods, pages 1-7, May 2016. doi: 10.1038/nmeth.3869.

Sarah Mollerup, Jens Friis-Nielsen, Lasse Vinner, Thomas Arn Hansen, Stine Raith Richter, Helena Fridholm, Jose Alejandro Romero Herrera, Ole Lund, Søren Brunak, Jose M G Izarzugaza, Tobias Mourier, Lars Peter Nielsen, and Anders Johannes Hansen. Propionibacterium acnes: Disease-Causing Agent or Common Contaminant? Detection in Diverse Patient Samples by Next-Generation Sequencing. Journal of clinical microbiology, 54(4):980-987, April 2016. doi: 10.1128/JCM.02723-15.

Ana Montalban-Arques, Philipp Wurm, Slave Trajanoski, Silvia Schauer, Sabine Kienesberger, Bettina Halwachs, Christoph Högenauer, Cord Langner, and Gregor Gorkiewicz. Propionibacterium acnes overabundance and natural killer group 2 member D system activation in corpus-dominant lymphocytic gastritis. The Journal of pathology, 240(4):425-436, December 2016. doi: 10.1002/path.4782.

C Mowat, C Williams, D Gillen, M Hossack, D Gilmour, A Carswell, A Wirz, T Preston, and K E McColl. Omeprazole, Helicobacter pylori status, and alterations in the intragastric milieu facilitating bacterial N-nitrosation. Gastroenterology, 119(2):339-347, August 2000. doi: 10.1053/gast.2000.9367.

Claudia Ota-Tsuzuki and Marcia Pinto Alves Mayer. Collagenase production and hemolytic activity related to $16 S$ rRNA variability among Parvimonas micra oral isolates. Anaerobe, 16(1):38-42, February 2010. doi: 10.1016/j.anaerobe.2009.03.008.

Chan Hyuk Park, Jae Gon Lee, A-Reum Lee, Chang Soo Eun, and Dong Soo Han. Network construction of gastric microbiome and organization of microbial modules associated with gastric carcinogenesis. Scientific Reports, 9(1):12444-9, August 2019.

Bryony N Parsons, Umer Z ljaz, Rosalinda D'Amore, Michael D Burkitt, Richard Eccles, Luca Lenzi, Carrie A Duckworth, Andrew R Moore, Laszlo Tiszlavicz, Andrea Varro, Neil Hall, and D Mark Pritchard. Comparison of the human gastric microbiota in hypochlorhydric states arising as a result of Helicobacter pyloriinduced atrophic gastritis, autoimmune atrophic gastritis and proton pump inhibitor use. PLoS Pathogens, 13(11):e1006653, November 2017. doi: 10.1371/ journal.ppat.1006653.
Price, Morgan N, Dehal, Paramvir S, and Arkin, Adam P. FastTree 2-approximately maximum-likelihood trees for large alignments. PLOS ONE, 5(3):e9490, March 2010. doi: 10.1371/journal.pone.0009490.

Rachel V Purcell, Martina Visnovska, Patrick J Biggs, Sebastian Schmeier, and Frank A Frizelle. Distinct gut microbiome patterns associate with consensus molecular subtypes of colorectal cancer. Scientific Reports, 7(1):11590, September 2017. doi: 10.1038/ s41598-017-11237-6.

Smruti Pushalkar, Xiaojie Ji, Yihong Li, Cherry Estilo, Ramanathan Yegnanarayana, Bhuvanesh Singh, Xin Li, and Deepak Saxena. Comparison of oral microbiota in tumor and non-tumor tissues of patients with oral squamous cell carcinoma. BMC Microbiology, 12(1): 144-15, July 2012. doi: 10.1186/1471-2180-12-144.

Rubayat Rahman, Akwi W Asombang, and Jamal A Ibdah. Characteristics of gastric cancer in Asia. World journal of gastroenterology: WJG, 20(16):4483-4490, April 2014. doi: $10.3748 /$ wjg.v20.i16.4483.

Cosmeri Rizzato, Javier Torres, Elena Kasamatsu, Margarita Camorlinga-Ponce, Maria Mercedes Bravo, Federico Canzian, and Ikuko Kato. Potential Role of Biofilm Formation in the Development of Digestive Tract Cancer With Special Reference to Helicobacter pylori Infection. Frontiers in Microbiology, 10:846, 2019. doi: 10.3389/fmicb.2019.00846.

Christian R Salazar, Jinghua Sun, Yihong Li, Fritz Francois, Patricia Corby, Guillermo Perez-Perez, Ananda Dasanayake, Zhiheng Pei, and Yu Chen. Association between selected oral pathogens and gastric precancerous lesions. PLOS ONE, 8(1):e51604, 2013. doi: 10.1371/journal.pone.0051604.

B K Sharma, I A Santana, E C Wood, R P Walt, M Pereira, P Noone, P L R Smith, C L Walters, and R E Pounder. Intragastric bacterial activity and nitrosation before, during, and after treatment with omeprazole. British Medical Journal, pages 1-3, March 1984. doi: 10.1136/ bmj.289.6447.717.

Irina I Shilnikova and Natalia V Dmitrieva. Evaluation of antibiotic susceptibility of Bacteroides, Prevotella and Fusobacterium species isolated from patients of the N. N. Blokhin Cancer Research Center, Moscow, Russia. Anaerobe, 31:15-18, February 2015. doi: 10.1016/j.anaerobe.2014.08.003.

Seiji Shiota, Varocha Mahachai, Ratha-korn Vilaichone, Thawee Ratanachu-ek, Lotay Tshering, Tomohisa Uchida, Osamu Matsunari, and Yoshio Yamaoka. Seroprevalence of Helicobacter pylori infection and gastric mucosal atrophy in Bhutan, a country with a high prevalence of gastric cancer. Journal of medical microbiology, 62(Pt 10):1571-1578, October 2013. doi: 10.1099/jmm.0.060905-0. 
bioRxiv preprint doi: https://doi.org/10.1101/2020.03.23.003426; this version posted April 18, 2020. The copyright holder for this preprint (which was not certified by peer review) is the author/funder, who has granted bioRxiv a license to display the preprint in perpetuity. It is made Revised manuscript available under aCC-BY-NC-ND 4.0 International license.

Lívia Jacovassi Tavares, Marlise Inêz Klein, Beatriz Helena Dias Panariello, Erica Dorigatti de Avila, and Ana Cláudia Pavarina. An in vitro model of Fusobacterium nucleatum and Porphyromonas gingivalis in single- and dual-species biofilms. Journal of periodontal \& implant science, 48(1):12-21, February 2018. doi: 10.5051/jpis.2018.48.1.12.

Veli-Jukka Uitto, Daniel Baillie, Qiang Wu, Renee Gendron, Daniel Grenier, Edward E Putnins, Arja Kanervo, and James D Firth. Fusobacterium nucleatum increases collagenase 3 production and migration of epithelial cells. Infection and Immunity, 73 (2):1171-1179, February 2005. doi: 10.1128/IAI.73.2. 1171-1179.2005.

A C M Veloo, G W Welling, and J E Degener. Antimicrobial susceptibility of clinically relevant Gram-positive anaerobic cocci collected over a three-year period in the Netherlands. Antimicrobial agents and chemotherapy, 55(3):1199-1203, March 2011. doi: 10.1128/AAC. 01771-09.

Jakob Wirbel, Paul Theodor Pyl, Ece Kartal, Konrad Zych, Alireza Kashani, Alessio Milanese, Jonas S Fleck, Anita Y Voigt, Albert Palleja, Ruby Ponnudurai, Shinichi Sunagawa, Luis Pedro Coelho, Petra SchrotzKing, Emily Vogtmann, Nina Habermann, Emma Niméus, Andrew M Thomas, Paolo Manghi, Sara Gandini, Davide Serrano, Sayaka Mizutani, Hirotsugu Shiroma, Satoshi Shiba, Tatsuhiro Shibata, Shinichi Yachida, Takuji Yamada, Levi Waldron, Alessio Naccarati, Nicola Segata, Rashmi Sinha, Cornelia M UIrich, Hermann Brenner, Manimozhiyan Arumugam, Peer Bork, and Georg Zeller. Meta-analysis of fecal metagenomes reveals global microbial signatures that are specific for colorectal cancer. Nature Medicine, 25(4):679-689, April 2019. doi: 10.1038/ s41591-019-0406-6.

Marta Wlodarska, Chengwei Luo, Raivo Kolde, Eva d'Hennezel, John W Annand, Cortney E Heim, Philipp Krastel, Esther K Schmitt, Abdifatah S Omar, Elizabeth A Creasey, Ashley L Garner, Sina Mohammadi, Daniel J O'Connell, Sahar Abubucker, Timothy D Arthur, Eric A Franzosa, Curtis Huttenhower, Leon O Murphy, Henry J Haiser, Hera Vlamakis, Jeffrey A Porter, and Ramnik J Xavier. Indoleacrylic Acid Produced by Commensal Peptostreptococcus Species Suppresses Inflammation. Cell host \& microbe, 22(1):25-37.e6, July 2017. doi: 10.1016/j.chom.2017. 06.007.

Jiao Wu, Qing Li, and Xiangsheng Fu. Fusobacterium nucleatum Contributes to the Carcinogenesis of Colorectal Cancer by Inducing Inflammation and Suppressing Host Immunity. Trans/ational oncology, 12(6):846-851, June 2019. doi: 10.1016/j.tranon.2019.03.003.
Kensuke Yamamura, Yoshifumi Baba, Keisuke Miyake, Kenichi Nakamura, Hironobu Shigaki, Kosuke Mima, Junji Kurashige, Takatsugu Ishimoto, Masaaki Iwatsuki, Yasuo Sakamoto, Yoichi Yamashita, Naoya Yoshida, Masayuki Watanabe, and Hideo Baba. Fusobacterium nucleatum in gastroenterological cancer: Evaluation of measurement methods using quantitative polymerase chain reaction and a literature review. Oncology letters, 14(6):6373-6378, December 2017. doi: 10.3892/ol.2017.7001.

Ines Yang, Sabrina Woltemate, M Blanca Piazuelo, Luis E Bravo, Maria Clara Yepez, Judith Romero-Gallo, Alberto G Delgado, Keith T Wilson, Richard M Peek, Pelayo Correa, Christine Josenhans, James G Fox, and Sebastian Suerbaum. Different gastric microbiota compositions in two human populations with high and low gastric cancer risk in Colombia. Scientific Reports, 6(1):18594-10, January 2016. doi: 10.1038/ srep18594.

Theresa Wan-Chen Yap, Han Ming Gan, Yin-Peng Lee, Alex Hwong-Ruey Leow, Ahmad Najib Azmi, Fritz Francois, Guillermo I Perez-Perez, Mun Fai Loke, Khean Lee Goh, and Jamuna Vadivelu. Helicobacter pylori Eradication Causes Perturbation of the Human Gut Microbiome in Young Adults. PLOS ONE, 11(3): e0151893, 2016. doi: 10.1371/journal.pone.0151893.

Guoqin Yu, Javier Torres, Nan Hu, Rafael MedranoGuzman, Roberto Herrera-Goepfert, Michael S Humphrys, Lemin Wang, Chaoyu Wang, Ti Ding, Jacques Ravel, Philip R Taylor, Christian C Abnet, and Alisa M Goldstein. Molecular Characterization of the Human Stomach Microbiota in Gastric Cancer Patients. Frontiers in cellular and infection microbiology, 7:302, 2017. doi: 10.3389/fcimb.2017.00302.

Jun Yu, Liuyang Zhao, Risheng Zhao, Xiaohang Long, Olabisi Oluwabukola Coker, and Joseph J.Y. Sung. The role of parvimonas micra in intestinal tumorigenesis in germ-free and conventional apcmin/+ mice. Journal of Clinical Oncology, 37(4_suppl):531-531, 2019. doi: 10.1200/JCO.2019.37.4\_suppl.531.

Georg Zeller, Julien Tap, Anita Y Voigt, Shinichi Sunagawa, Jens Roat Kultima, Paul I Costea, Aurelien Amiot, Jürgen Böhm, Francesco Brunetti, Nina Habermann, Rajna Hercog, Moritz Koch, Alain Luciani, Daniel R Mende, Martin A Schneider, Petra SchrotzKing, Christophe Tournigand, Jeanne Tran Van Nhieu, Takuji Yamada, Jürgen Zimmermann, Vladimir Benes, Matthias Kloor, Cornelia M Ulrich, Magnus von Knebel Doeberitz, Iradj Sobhani, and Peer Bork. Potential of fecal microbiota for early-stage detection of colorectal cancer. Molecular systems biology, 10 (11):766-766, November 2014. doi: 10.15252/msb. 20145645. 